\title{
Giant enhancement of spin detection sensitivity in (Ga,Mn)As/GaAs Esaki diodes
}

\author{
Junichi Shiogai, ${ }^{1,}{ }^{*}$ Mariusz Ciorga,${ }^{2, \dagger}$ Martin Utz, ${ }^{2}$ Dieter Schuh,${ }^{2}$ Makoto Kohda, ${ }^{1}$ Dominique Bougeard, ${ }^{2}$ \\ Tsutomu Nojima, ${ }^{3}$ Junsaku Nitta, ${ }^{1}$ and Dieter Weiss ${ }^{2}$ \\ ${ }^{1}$ Department of Materials Science, Tohoku University, 980-8579 Sendai, Miyagi, Japan \\ ${ }^{2}$ Institute of Experimental and Applied Physics, University of Regensburg, D-93040 Regensburg, Germany \\ ${ }^{3}$ Institute of Materials Research, Tohoku University, 980-8577 Sendai, Miyagi, Japan \\ (Received 22 October 2013; revised manuscript received 22 January 2014; published 21 February 2014)
}

\begin{abstract}
We investigate the correlation between spin signals measured in three-terminal (3T) geometry by the Hanle effect and the spin accumulation generated in a semiconductor channel in a lateral (Ga,Mn)As/GaAs Esaki diode device. We systematically compare measurements using a 3T configuration, probing spin accumulation directly beneath the injecting contact, with results from nonlocal measurements, where solely spin accumulation in the GaAs channel is probed. We find that the spin signal detected in the $3 \mathrm{~T}$ configuration is dominated by a bias-dependent spin detection sensitivity, which in turn is strongly correlated with charge-transport properties of the junction. This results in a particularly strong enhancement of the detected spin signal in a region of increased differential resistance. We find additionally that two-step tunneling via localized states in the gap of (Ga,Mn)As does not compromise spin injection into the semiconductor conduction band.
\end{abstract}

DOI: 10.1103/PhysRevB.89.081307

All-electrical generation, manipulation, and detection of spin-polarized electrons in semiconductors are key prerequisites for the realization of spin-based electronic devices [1,2]. In recent years there has been considerable progress in understanding the basic processes governing electrical spin injection from a ferromagnet (FM) into a semiconductor (SC), with numerous theoretical and experimental contributions [3-10]. Despite this, the large spin signals [11], measured particularly in Si- and Ge-based devices [12-14], withstand so far straightforward explanation and go well beyond (i.e., orders of magnitude) the commonly accepted standard model of spin injection [3,4]. These large values have been found using a three-terminal (3T) method of spin detection, with one single magnetic contact used to inject and detect spin accumulation. An initially proposed model explained the observed giant spin signal enhancement in terms of an enhanced spin accumulation generated solely in states localized at the FM-SC interface [11]. The enhancement is then driven by the large resistance between localized states (LS) and the SC channel, due to a depletion zone in the interface region. Such a scenario would however impede actual spin injection into the SC channel itself $[11,15]$, calling into question also the applicability of the 3T method to detect spin accumulation in the SC channel. This constraint was relaxed in an extension of the LS model [16], allowing for direct tunneling of electrons between the FM and SC conduction band, suggesting that direct band-to-band tunneling and double-step tunneling, involving LS, occur simultaneously [16]. It was shown in some experiments that the spin signal can be enhanced even in the absence of a depletion region $[14,17]$.

What has been missing so far is a systematic comparison between $3 \mathrm{~T}$ and nonlocal (NL) measurements in the same devices, showing large signals in the 3T configuration. Such a comparison is essentially needed in order to unambiguously establish a correlation between the 3T signal and the actual spin

\footnotetext{
*j.shiogai@s.tohoku.ac.jp

†mariusz.ciorga@physik.uni-regensburg.de
}

PACS number(s): 72.25.Hg, 72.25.Mk, 75.50.Pp

accumulation in the channel. What also has been overlooked in the recent discussion is the detection sensitivity of spin detecting contacts $[18,19]$. It was shown theoretically by Chantis and Smith [18] and observed experimentally by Crooker et al. [19] that a current-biased spin detector has its sensitivity dramatically changed compared to a nonbiased case. This makes charge transport through the detecting contact, in particular in the presence of any nonlinearity, a very important factor. Because one uses a biased contact as spin detector in 3T configuration, these effects should be taken into account for analyzing the measured signals.

In this Rapid Communication we employ a lateral $(\mathrm{Ga}, \mathrm{Mn}) \mathrm{As} / \mathrm{GaAs}$ spin injection device as a test bed [7,2024] to investigate the effects described above. The use of Esaki diodes as spin sensitive contacts gives us the unique opportunity to tune the relative contribution of direct and two-step tunneling via LS in the gap of $(\mathrm{Ga}, \mathrm{Mn}) \mathrm{As}$ in a single device by simply changing the bias across the junction [25]. We show that (i) tunneling through the LS, does not affect spin accumulation in the conduction band of GaAs and (ii) the detection sensitivity is strongly affected by the nonlinearity of the current-voltage $(I-V)$ characteristic of the contact. A schematic of a typical spin injection device is depicted in Fig. 1(a). The device is patterned into a $50-\mu \mathrm{m}$-wide [110] oriented mesa by standard photolithography and wet chemical etching using diluted acetic acid: $\mathrm{H}_{2} \mathrm{O}_{2}: \mathrm{H}_{2} \mathrm{O}$ solution. The corresponding wafer consists of a semi-insulating GaAs (001) substrate, a 300-nm GaAs buffer layer, a 500-nm AlGaAs/GaAs superlattice, $0.8-\mu \mathrm{m} n$-GaAs, $0.2-\mu \mathrm{m} n^{+}$-GaAs, a $15-\mathrm{nm} n^{+} \rightarrow n^{++}$-GaAs transition layer, 8.0-nm $n^{++}-\mathrm{GaAs}$, a 2.2-nm AlGaAs diffusion barrier, and 50-nm (Ga,Mn)As. The doping concentrations of the GaAs layers are $n=2 \times 10^{16} \mathrm{~cm}^{-3}, n^{+}=6 \times 10^{16} \mathrm{~cm}^{-3}$, and $n^{++}=6 \times 10^{18} \mathrm{~cm}^{-3}$, respectively. Ferromagnetic contacts, aligned along [1110], were defined by electron-beam lithography, $\mathrm{Au} / \mathrm{Ti}$ evaporation, and liftoff. Contact 2 is $4-\mu \mathrm{m}$ and the other contacts (3-6) are $0.5-\mu \mathrm{m}$-wide. The center-to-center spacing between neighboring contacts is $5 \mu \mathrm{m}$, and $L$ is the distance between the injector and detector. Large reference 

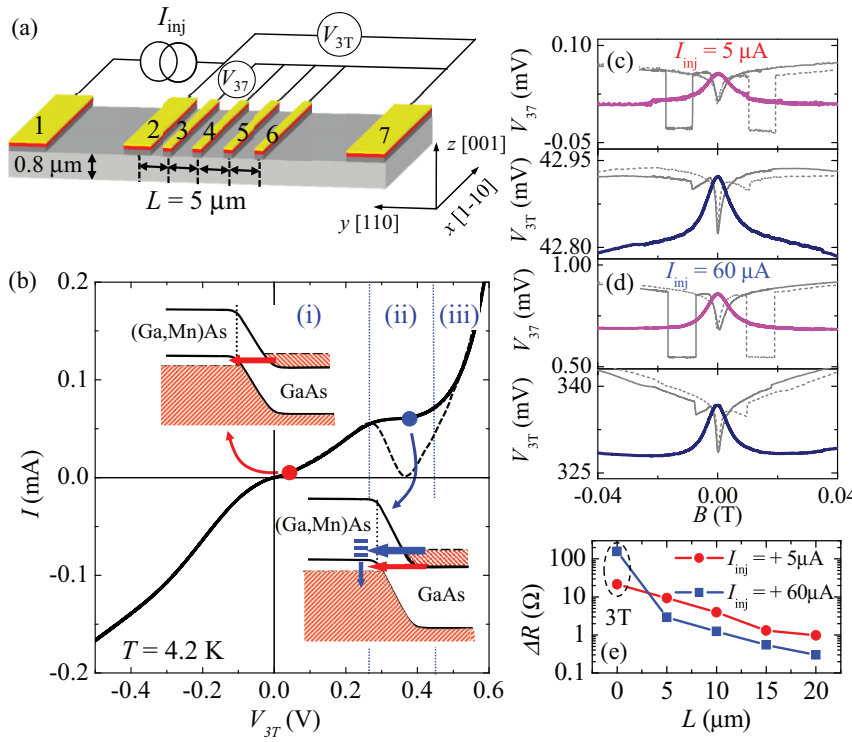

FIG. 1. (Color online) (a) Multiterminal spin injection device for three-terminal (3T) and nonlocal (NL) detection. (b) Current-voltage $(I-V)$ characteristic of spin Esaki contact 2. The dashed line shows schematically the $I-V$ characteristic of an ideal Esaki diode in the absence of excess current. Insets: Direct (upper) and indirect (lower) tunneling processes. (c) NL (upper panel) and 3T (lower panel) voltages in the regime of direct tunneling $\left(I_{\mathrm{inj}}=5 \mu \mathrm{A}\right)$. Colored traces are plotted as a function of out-of-plane field $B_{\mathrm{z}}$, while the thin gray lines are up- and down-sweeps of the in-plane field $B_{\mathrm{x}}$. (d) As (c), but for $I_{\text {inj }}=60 \mu \mathrm{A}$, i.e., in the excess current regime. (e) The Hanle signals $\Delta R_{\mathrm{nl}(3 \mathrm{~T})}^{\mathrm{Hanle}}=\Delta V_{\mathrm{nl}(3 \mathrm{~T})}^{\mathrm{Hanl}} / I_{\mathrm{inj}}$ for both regimes as a function of $L$. The signals obtained by $3 \mathrm{~T}$ configuration are plotted at $L=0$ $\mu \mathrm{m}$. All measurements are performed at $T=4.2 \mathrm{~K}$.

contacts 1 and $7\left(150 \times 150 \mu \mathrm{m}^{2}\right)$ were defined at the end of the mesa by photolithography and $\mathrm{Au} / \mathrm{Ti}$ evaporation. Finally, the $(\mathrm{Ga}, \mathrm{Mn}) \mathrm{As}$ and the highly doped GaAs layers were removed between the contacts by reactive ion etching to confine the current flow within the low-doped GaAs channel. Contact 2 was usually used as injector; the others were used as detectors. A nonequilibrium spin accumulation generated underneath the injector by driving a current $I_{\text {inj }}$ between the FM injector and reference contact 1 can then be probed either nonlocally or using the $3 \mathrm{~T}$ method. All measurements were performed at $T=4.2 \mathrm{~K}$.

Let us first discuss the $I-V$ characteristic of contact 2 , taken in the 3T configuration and shown in Fig. 1(b). The current through an Esaki diode consists of different contributions from (i) direct tunneling between the valence band of $p-(\mathrm{Ga}, \mathrm{Mn}) \mathrm{As}$ and the conduction band of $n$-GaAs, (ii) tunneling through LS in the band gap (constituting the so-called excess current [25]), and (iii) thermal transport across the built-in potential. Component (iii), not interesting for spin injection, is dominating at high forward bias. At reverse bias and for small forward bias, component (i) dominates the current as electrons tunnel from $(\mathrm{Ga}, \mathrm{Mn}) \mathrm{As}$ into $\mathrm{GaAs}$ or in the opposite direction. The latter case is schematically shown in the upper inset of Fig. 1(b). A further increase of $V_{3 \mathrm{~T}}$ removes the overlap of the bands, suppressing component (i). For an ideal Esaki diode this would lead to a vanishing current [see the dashed curve in
Fig. 1(b)]. In real devices, however, component (ii) dominates in this regime and is responsible for a nonzero tunnel current [25]. The importance of this process is manifested by a very shallow Esaki dip at about $0.4 \mathrm{~V}$ in Fig. 1(b), observed in all our $(\mathrm{Ga}, \mathrm{Mn}) \mathrm{As} / \mathrm{GaAs}$ junctions and indicating the presence of a large excess current. This is not surprising as $(\mathrm{Ga}, \mathrm{Mn}) \mathrm{As}$, grown at low temperatures, contains a high density of LS in the gap [26-28] supporting two-step (or multistep) tunneling. This situation is depicted in the lower inset of Fig. 1(b), showing electrons tunneling from the conduction band either into LS or directly into the valence band. For our further discussion it is important to note that the $I-V$ characteristic of the Esaki diode, while nearly linear in regime (i), becomes highly nonlinear in regime (ii). Thus, by tuning $V_{3 \mathrm{~T}}$ between the red and the blue point in Fig. 1(b), both the ratio of direct and indirect tunneling currents and the degree of nonlinearity of the $I-V$ characteristics are widely changed.

In our NL measurements the four $0.5-\mu \mathrm{m}$-wide contacts 3-6 are used as nonlocal spin detectors probing pure spin currents flowing from the injector toward the detectors. According to the standard drift-diffusion model, the spin accumulation at the injection point, $\mu_{\mathrm{s}}(0)=-P_{\mathrm{inj}} j r_{\mathrm{s}}^{\mathrm{ch}}=-P_{\mathrm{inj}} j \rho_{\mathrm{N}} \lambda_{\mathrm{sf}}$, where $\rho_{\mathrm{N}}$ and $\lambda_{\mathrm{sf}}$ are the resistivity and the spin-diffusion length of the GaAs channels, respectively, and $r_{\mathrm{s}}^{\mathrm{ch}}$ is the effective spin resistance of the channel. It gives rise to the following NL voltage at the detection point $y=L[4-7,29]$ :

$$
\begin{aligned}
V^{s}(L) & =-P_{\operatorname{det}} \mu_{\mathrm{s}}(L) / 2 \\
& = \pm\left(P_{\text {inj }} P_{\operatorname{det}} I_{\text {inj }} \lambda_{\mathrm{sf}} \rho_{\mathrm{N}} / 2 S\right) \exp \left(-L / \lambda_{\mathrm{sf}}\right),
\end{aligned}
$$

where $I_{\text {inj }}$ is the spin injection current, $S$ is the cross-sectional area of the nonmagnetic channel, and $P_{\text {inj(det) }}$ is the tunneling spin polarization (TSP) of the injector (detector). The $+(-)$ sign stands for the parallel (antiparallel) magnetization alignment of the injector and detector. The magnetization configuration is switched between parallel and antiparallel by sweeping an in-plane magnetic field $B_{\mathrm{x}}$. The switching results in a voltage jump $\Delta V_{\mathrm{nl}}^{\mathrm{SV}}=2 V^{\mathrm{s}}(L)$ which is a direct measure of the generated $\mu_{\mathrm{s}}(L)$. Alternatively, the Hanle effect is used. An out-of-plane magnetic field $B_{\mathrm{z}}$ causes a precession of the in-plane electron spins, which results in a decay of the Hanle amplitude $\Delta V_{\mathrm{nl}}^{\text {Hanle }}=V^{\mathrm{s}}(L)$.

Spin detection in the $3 \mathrm{~T}$ configuration relies on the fact that spins accumulated at the FM-SC interface increase the voltage drop across the junction $[11,16]$. The spin-related contribution to $V_{3 \mathrm{~T}}$ is typically described by Eq. (1) assuming $L=0$ and $P_{\mathrm{inj}}=P_{\mathrm{det}}$ and can be determined by Hanle measurements. The suppression of spin accumulation at finite $B_{\mathrm{Z}}$ results in a reduction of the measured $V_{3 \mathrm{~T}}$ with the signal amplitude $\Delta V_{3 \mathrm{~T}}^{\text {Hanle }}=V^{\mathrm{s}}(L=0)$.

Spin signals measured in the regime of (i) direct tunneling and (ii) indirect tunneling are shown in Figs. 1(c) and 1(d), respectively. Clear nonlocal spin valve (NLSV) and Hanle curves, observed for both bias regimes and for all NL detectors, indicate spin accumulation in the conduction band of GaAs. Figure 1(e) shows the distance dependence of the Hanle signals $\Delta R_{\mathrm{nl}(3 \mathrm{~T})}^{\text {Hanle }}=\Delta V_{\mathrm{nl}(3 \mathrm{~T})}^{\text {Hanle }} / I_{\text {inj }}$ measured in $3 \mathrm{~T}(L=0)$ and NL configurations. From this dependence we determine the spin-diffusion length as $\lambda_{\mathrm{sf}}=6.0 \mu \mathrm{m}$; using Eq. (1) we extract $P_{\text {inj }}=0.641$ at $V_{3 \mathrm{~T}}=0.043 \mathrm{~V}$ and $P_{\text {inj }}=0.194$ 


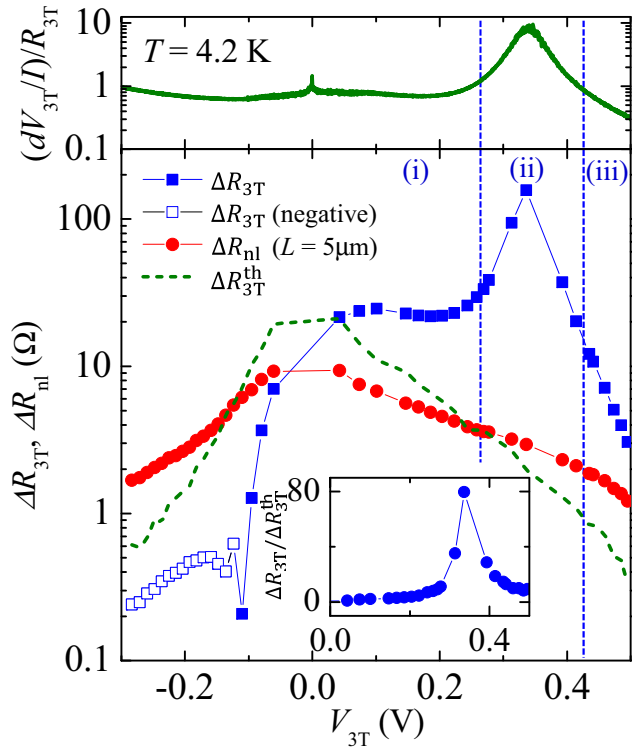

FIG. 2. (Color online) Bottom panel: 3T-Hanle signal $\Delta R_{3 \mathrm{~T}}^{\text {Hanle }}=$ $\Delta V_{3 \mathrm{~T}}^{\text {Hanle }} / I_{\mathrm{inj}}$, detected at contact 2 and NL-Hanle signal $\Delta R_{\mathrm{nl}}^{\text {Hanle }}=$ $\Delta V_{\mathrm{nl}}^{\text {Hanle }} / I_{\mathrm{inj}}$, measured at contact 3 and plotted as a function of bias voltage $V_{3 \mathrm{~T}}$ at $T=4.2 \mathrm{~K}$. Open symbols indicate a negative sign in $\Delta R_{3 \mathrm{~T}}^{\text {Hanle }}$. The dashed line shows the expected value for $\Delta R_{3 \mathrm{~T}}^{\text {th }}$ calculated from Eq. (1) for $L=0$ using parameters extracted from NL measurements. Inset: Enhancement factor defined as $\Delta R_{3 \mathrm{~T}}^{\text {Hanle }} / \Delta R_{3 \mathrm{~T}}^{\mathrm{th}}$. Top panel: the ratio of differential resistance $d V_{3 \mathrm{~T}} / d I$ and junction resistance $R_{3 \mathrm{~T}}=V_{3 \mathrm{~T}} / I$ calculated from the $I-V$ characteristic shown in Fig. 1.

at $V_{3 \mathrm{~T}}=0.336 \mathrm{~V}$ in good agreement with our previous work [7,30]. Whereas the NL signal is larger in the direct tunneling regime $\left(I_{\mathrm{inj}}=5 \mu \mathrm{A}\right)$, the $3 \mathrm{~T}$ signal, in contrast, increases from $\Delta R_{3 \mathrm{~T}}^{\mathrm{Hanle}}=21 \Omega$ in the low bias regime to $157 \Omega$ in the impurity-assisted tunneling regime $\left(I_{\text {inj }}=60\right.$ $\mu \mathrm{A})$. Using Eq. (1) and the parameters extracted from NL measurements we can estimate the expected Hanle signal: For the direct tunneling regime $\left(V_{3 \mathrm{~T}}=0.043 \mathrm{~V}\right)$ we calculate $\Delta R_{3 \mathrm{~T}}^{\text {Hanle }}=20 \Omega$, while we obtain $\Delta R_{3 \mathrm{~T}}^{\text {Hanle }} \approx 1.8 \Omega$ for the indirect tunneling $\left(V_{3 \mathrm{~T}}=0.336 \mathrm{~V}\right)$. While calculated and measured Hanle signals are nearly the same in the regime (i), where the $I-V$ characteristic is nearly linear, they differ by about two orders of magnitude in the regime (ii), i.e., when the $I-V$ characteristic becomes nonlinear.

To investigate this discrepancy in more detail we systematically studied the dependence of the spin signals on the bias voltage and current across the injector. The results are summarized in Fig. 2, where we plot both $\Delta R_{\mathrm{nl}}^{\text {Hanle }}$ and $\Delta R_{3 \mathrm{~T}}^{\mathrm{Hanle}}$ as a function of $V_{3 \mathrm{~T}}$. The NL resistance $\Delta R_{\mathrm{nl}}^{\mathrm{Hanle}}$ decreases monotonically with increasing bias for both bias polarities, ascribed to a decrease of $P_{\text {inj }}$ [7]. The behavior of the 3T Hanle signal $\Delta R_{3 \mathrm{~T}}^{\mathrm{Hanl}}$ is strikingly different from the theoretical prediction $\Delta R_{3 \mathrm{~T}}^{\mathrm{th}}=P^{2} \lambda_{\mathrm{sf}} \rho_{\mathrm{N}} / 2 S$, which is plotted as a dashed curve in Fig. 2 [31]. Contrary to the latter, $\Delta R_{3 \mathrm{~T}}^{\mathrm{Hanle}}$ slowly increases for positive bias, reaches a plateau, and then rises again to reach a maximum at the Esaki dip. A further increase of the voltage rapidly decreases the signal. For reverse bias the signal rapidly drops to zero before changing its sign at $V_{3 \mathrm{~T}}=-0.1 \mathrm{~V}$. The behavior at low positive and low negative bias resembles well NL experiments on the Fe/GaAs system with a biased detector, interpreted in terms of bias dependence of the detector sensitivity [18,19]. The sensitivity is defined as a change in a voltage drop $\Delta V$ across the biased FM-SC interface as a result of spin accumulation $\Delta \mu_{\mathrm{s}}$ generated in the SC. Its bias dependence can be quite different from that of $P_{\text {inj(det) }}$ and stems from the dependence of the density of spinpolarized carriers underneath the detector on the electric field in the channel and at the interface. As a result the spin signal is expected to be enhanced for spin extraction $\left(I_{\text {inj }}>0\right)$ and suppressed for spin injection $\left(I_{\text {inj }}<0\right)$ cases. This is exactly what we observe in the experiments as $\Delta R_{3 \mathrm{~T}}^{\mathrm{Hanle}}>\Delta R_{3 \mathrm{~T}}^{\mathrm{th}}$ for the former and $\Delta R_{3 \mathrm{~T}}^{\mathrm{Hanle}}<\Delta R_{3 \mathrm{~T}}^{\mathrm{th}}$ for the latter (see Fig. 2). The sign change for $V_{3 \mathrm{~T}}<-0.1 \mathrm{~V}$ we also attribute to the electric-field dependent detection sensitivity [32]. Because the 3T signal is proportional to $P_{\text {inj }} P_{\text {det }}=(\mathrm{TSP})^{2}$ [see Eq. (1)] its sign reversal cannot be ascribed to a sign reversal of TSP, caused, e.g., by resonant states of the interfacial minority spin band [33-35].

Let us now discuss the huge enhancement of the local signal in regime (ii), where the tunneling current is dominated by the excess current. Because we do not observe either enhancement or suppression in the NL signal, one can conclude that the spin accumulation in the channel is not affected by the excess current. There are then two possible mechanisms which can account for the enhancement of the 3T signal. The first one involves spin injection into LS with a higher spin effective resistance $r_{\mathrm{s}}^{\text {ls }}$ than the one in the channel $r_{\mathrm{s}}^{\mathrm{ch}}$ [11]. This would result in a higher spin accumulation underneath the injector and would thus dominate the measured $\Delta R_{3 \mathrm{~T}}^{\mathrm{Hanle}}$ without changing the spin current in the channel. A second possible mechanism is based on an increased sensitivity of spin detection in the highly nonlinear region (ii). It can be explained as follows. Consider the voltage drop across the junction $V_{3 \mathrm{~T}}$ in the presence of the constant injection current $I$. It contains the contribution $V^{\mathrm{s}}=-\left(P_{\mathrm{det}} / 2\right) \mu_{\mathrm{s}}$ stemming from the generated spin accumulation $\mu_{\mathrm{s}}$, and for $I>0$ it can be written as $V_{3 \mathrm{~T}}\left(\mu_{\mathrm{s}}\right)=I R\left(V_{3 \mathrm{~T}}\right)+\left(P_{\mathrm{det}} / 2\right)\left|\mu_{\mathrm{s}}\right|$, taking into account that the interface resistance $R_{3 \mathrm{~T}}=R\left(V_{3 \mathrm{~T}}\right)$ is also voltage dependent. In the Hanle experiments the spin accumulation is reduced by $\Delta \mu_{\mathrm{s}}$ due to the applied $B_{\mathrm{Z}}$, resulting in spin precession and dephasing. A condition of the constant current requires readjustment of the voltage across the junction by $\Delta V_{3 \mathrm{~T}}$ if the spin accumulation changes by $\Delta \mu_{\mathrm{s}}$. This adjustment, which constitutes the detection sensitivity addressed above, is readily obtained by taking a derivative of the above expression with respect to $\mu_{\mathrm{s}}$ and results in $\Delta V_{3 \mathrm{~T}}=\left(d V_{3 \mathrm{~T}} / d I\right) /\left(V_{3 \mathrm{~T}} / I\right) P_{\mathrm{det}} \Delta \mu_{\mathrm{s}} / 2$, in agreement with the expression derived in Ref. [18]. This means that the spin detection sensitivity is amplified by the ratio of the differential resistance and the interface resistance, a measure of nonlinearity of the $I-V$ curve. The $3 \mathrm{~T}$ Hanle signal $\Delta V_{3 \mathrm{~T}}^{\mathrm{Hanle}}$, measured as the voltage change $\Delta V_{3 \mathrm{~T}}$ due to full depolarization of the spin accumulation, is then expected to be proportional to that ratio. This proportionality can be seen in Fig. 2 by comparing the $\Delta R_{3 \mathrm{~T}}^{\mathrm{Hanle}}$ signal with $\left(d V_{3 \mathrm{~T}} / d I\right) /\left(V_{3 \mathrm{~T}} / I\right)$ (top panel), calculated from the $I-V$ curve (see Fig. 1). The 3T signal is clearly enhanced in the region of high nonlinearity.

To disentangle these two contributions we performed SV measurements with the setup shown schematically in 

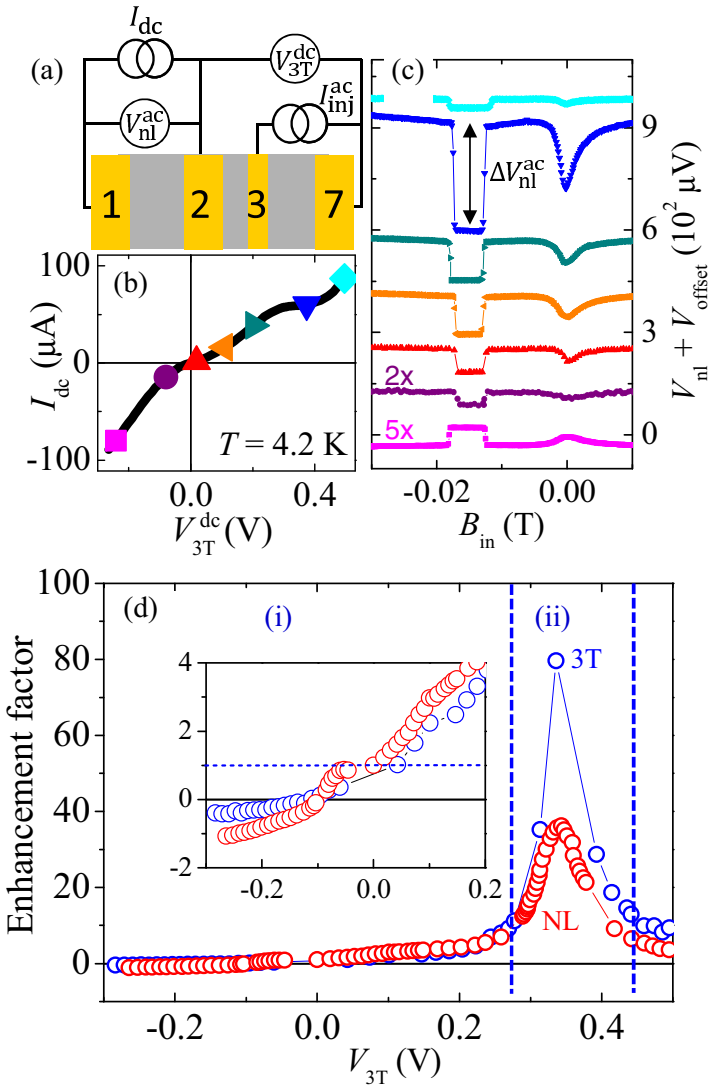

FIG. 3. (Color online) (a) Schematic of the circuit used for ac and dc measurements to extract the bias-dependent detection sensitivity. (b) Corresponding $I-V$ curve of contact 2. (c) SV signal traces measured at $\mathrm{NL}$ detector 2, with spin accumulation generated by contact 3 using $I_{\mathrm{ac}}=4.7 \mu \mathrm{A}$, and dc bias voltages $V_{3 \mathrm{~T}}^{\mathrm{dc}}$ as marked in (b). Curves are shifted for clarity. (d) Enhancement (suppression) of the NL and 3T signals by applied dc bias. Inset: Data from low-bias measurements, i.e., in the regime of direct tunneling.

Fig. 3(a), allowing us to directly measure the spin detection sensitivity [19]. Now, contact 2 serves as a biased NL detector of the spin accumulation generated in the GaAs channel by applying a small ac current bias with frequency $17 \mathrm{~Hz}$ to contact 3 . The NL voltage $V_{\mathrm{nl}}^{\text {ac }}$ is then measured as a function of a dc current bias applied to contact 2, used to tune $V_{3 \mathrm{~T}}^{\mathrm{dc}}$. In Fig. 3(c) we show the NLSV signal $V_{\mathrm{nl}}^{\text {ac }}$, at different values of $V_{3 \mathrm{~T}}^{\mathrm{dc}}$ marked in Fig. 3(b). The SV amplitude $\Delta V_{\mathrm{nl}}^{\mathrm{ac}}$, which is now a direct measure of the spin detection sensitivity, strongly depends on the applied $I_{\mathrm{dc}}$ in a similar manner as the 3T signal: a suppression and sign reversal is observed at negative bias while a strong amplification is observed at the Esaki dip.

In Fig. 3(d) we compare the bias-dependent enhancement of the spin signals observed in both configurations, i.e., $\Delta R_{3 \mathrm{~T}}^{\mathrm{Hanle}}$ in Fig. 2 and $\Delta V_{\mathrm{nl}}^{\mathrm{ac}}$ in Fig. 3(c), introducing an enhancement factor. In the case of the biased NL detector it is calculated as $\Delta V_{\mathrm{nl}}^{\mathrm{ac}} / \Delta V_{\mathrm{nl}}^{\mathrm{ac}}\left(I_{\mathrm{dc}}=0\right) \times P_{\mathrm{det}}\left(I_{\mathrm{dc}}=0\right) / P_{\mathrm{det}}$ and plotted as red circles in Fig. 3(d). Here we take into account that $P_{\text {det }}$ decreases with increasing bias current $I_{\mathrm{dc}}$. In 3T case the enhancement factor is defined as $\Delta R_{3 \mathrm{~T}}^{\mathrm{Hanl}} / \Delta R_{3 \mathrm{~T}}^{\mathrm{th}}$, i.e., the ratio of the blue and the green dashed traces in Fig. 2. In the regime of direct tunneling (i), plotted in the inset of Fig. 3(d), signals in both configurations show good qualitative and quantitative agreement, i.e., enhancement for $V_{3 \mathrm{~T}}>0$ and suppression and sign reversal for $V_{3 \mathrm{~T}}<0$, due to drift effects. This behavior is fully consistent with the results of Ref. [19]. As a result of nonlinearity in the region (ii) the detection sensitivity is further enhanced [18], reaching a factor of 36 . As the nonlinear contribution to the enhancement reaches the factor of 8 at the maximum (see the top panel in Fig. 2), we estimate the drift-induced contribution to be about 4 . This value is consistent with the enhancement in the low bias regime [see the inset in Fig. 3(d)]. The enhancement of the $3 \mathrm{~T}$ signal in the nonlinear region is, however, still two times higher, suggesting that the excess current generates also spin accumulation in gap states that contributes to the signal. We conclude, therefore, that the enhanced 3T signal, although having the contribution from LS, originates predominantly from the increased sensitivity to detect a conduction-band spin accumulation. This enhancement is strongly correlated with charge transport through the interface, namely, the nonlinearity of the $I-V$ characteristic of the junction.

In summary, we studied the correlation between $3 \mathrm{~T}$ spin signal and spin accumulation in the semiconductor channel probed in NL geometry. Our first fully comparative 3T- and NL-Hanle experiments show that tunneling through LS does not affect spin injection into the conduction band of a SC channel and that the $3 \mathrm{~T}$ method can be used to detect spin accumulation in the channel. One has to be very careful, however, while extracting the actual magnitude of the generated spin accumulation, as the measured signal is dominated by the bias-dependent sensitivity of spin detection [18,19]. As a result, Eq. (1) can no longer be used to describe the measured spin signal when the detector is biased, as in the case of the $3 \mathrm{~T}$ method. This aspect of the 3T spin detection was hitherto not taken into account, although some experiments on $\mathrm{Si}$ devices show correlation between measured spin signals and tunnel resistance [36,37] or differential resistance [38] of the junction. Although our experiments were conducted on spin Esaki diode devices, we find the results are quite general. Especially, the possibility to amplify the tiny NL spin signals by engineering a tunnel barrier in the detector in a way that it shows a high $\left(d V_{3 \mathrm{~T}} / d I\right) /\left(V_{3 \mathrm{~T}} / I\right)$ ratio can be of significant importance for the development of future spintronic devices.

We thank H. Jaffrès and J. Fabian for very fruitful discussions. This work was partly supported by the German Science Foundation [Deutsche Forschungsgemeinschaft (DFG)] via SFB 689, the Japan-Germany Strategic International Cooperative Program (Joint Research Type) from Japan Science and Technology Agency and DFG (FOR 1483), and Grants-in-Aid from JSPS 22226001 and 24684019.
[1] S. Datta and B. Das, Appl. Phys. Lett. 56, 665 (1990).
[2] I. Žutić, J. Fabian, and S. D. Sarma, Rev. Mod. Phys. 76, 323 (2004). 
[3] A. Fert and H. Jaffrès, Phys. Rev. B 64, 184420 (2001).

[4] J. Fabian, A. Matos-Abiague, C. Ertler, P. Stano, and I. Žutić, Acta Physica Slovaca 57, 565 (2007).

[5] X. Lou, C. Adelmann, S. A. Crooker, E. S. Garlid, J. Zhang, K. S. M. Reddy, S. D. Flexner, C. J. Palmstrøm, and Crowell, Nat. Phys. 3, 197 (2007).

[6] N. Tombros, C. Jozsa, M. Popinciuc, H. T. Jonkman, and B. J. van Wees, Nature (London) 448, 571 (2007).

[7] M. Ciorga, A. Einwanger, U. Wurstbauer, D. Schuh, W. Wegscheider, and D. Weiss, Phys. Rev. B 79, 165321 (2009).

[8] T. Sasaki, T. Oikawa, T. Suzuki, M. Shiraishi, Y. Suzuki, and K. Tagami, Appl. Phys. Express 2, 053003 (2009).

[9] Y. Zhou, W. Han, L. T. Chang, F. Xiu, M. Wang, M. Oehme, I. A. Fischer, J. Schulze, R. K. Kawakami, and K. L. Wang, Phys. Rev. B 84, 125323 (2011).

[10] X. Lou, C. Adelmann, M. Furis, S. A. Crooker, C. J. Palmstrøm, and P. A. Crowell, Phys. Rev. Lett. 96, 176603 (2006).

[11] M. Tran, H. Jaffrès, C. Deranlot, J. M. George, A. Fert, A. Miard, and A. Lemaître, Phys. Rev. Lett. 102, 036601 (2009).

[12] H. Saito, S. Watanabe, Y. Mineno, S. Sharma, R. Jansen, S. Yuasa, and K. Ando, Solid State Commun. 151, 1159 (2011).

[13] K. R. Jeon, B. C. Min, Y. H. Jo, H. S. Lee, I. J. Shin, C. Y. Park, S. Y. Park, and S. C. Shin, Phys. Rev. B 84, 165315 (2011).

[14] S. P. Dash, S. Sharma, R. S. Patel, M. P. de Jong, and R. Jansen, Nature (London) 462, 491 (2009).

[15] A. Jain, J. C. Rojas-Sanchez, M. Cubukcu, J. Peiro, J. C. LeBreton, E. Prestat, C. Vergnaud, L. Louahadj, C. Portemont, C. Ducruet, V. Baltz, A. Barski, P. Bayle-Guillemaud, L. Vila, J. P. Attané, E. Augendre, G. Desfonds, S. Gambarelli, H. Jaffrès, J. M. George, and M. Jamet, Phys. Rev. Lett. 109, 106603 (2012).

[16] R. Jansen, A. M. Deac, H. Saito, and S. Yuasa, Phys. Rev. B 85, 134420 (2012).

[17] S. Iba, H. Saito, A. Spiesser, S. Watanabe, R. Jansen, S. Yuasa, and K. Ando, Appl. Phys. Express 5, 023003 (2012).

[18] A. N. Chantis and D. L. Smith, Phys. Rev. B 78, 235317 (2008).

[19] S. A. Crooker, E. S. Garlid, A. N. Chantis, D. L. Smith, K. S. M. Reddy, Q. O. Hu, T. Kondo, C. J. Palmstrøm, and P. A. Crowell, Phys. Rev. B 80, 041305(R) (2009).

[20] E. Johnston-Halperin, D. Lofgreen, R. K. Kawakami, D. K. Young, L. Coldren, A. C. Gossard, and D. D. Awschalom, Phys. Rev. B 65, 041306 (2002).

[21] M. Kohda, Y. Ohno, K. Takamura, F. Matsukura, and H. Ohno, Jpn. J. Appl. Phys. 40, L1274 (2001).

[22] P. V. Dorpe, Z. Liu, W. V. Roy, V. F. Motsnyi, M. Sawicki, G. Borghs, and J. D. Boeck, Appl. Phys. Lett. 84, 3495 (2004).
[23] J. Shiogai, M. Ciorga, M. Utz, D. Schuh, T. Arakawa, M. Kohda, K. Kobayashi, T. Ono, W. Wegscheider, D. Weiss, and J. Nitta, Appl. Phys. Lett. 101, 212402 (2012).

[24] B. Endres, M. Ciorga, M. Schmid, M. Utz, D. Bougeard, D. Weiss, G. Bayreuther, and C. H. Back, Nat. Comm. 4, 2068 (2013).

[25] A. G. Chynoweth, W. L. Feldmann, and R. A. Logan, Phys. Rev. 121, 684 (1961).

[26] R. M. Feenstra, J. M. Woodall, and G. D. Pettit, Phys. Rev. Lett. 71, 1176 (1993).

[27] T. Tsuruoka, N. Tachikawa, S. Ushioda, F. Matsukura, K. Takamura, and H. Ohno, Appl. Phys. Lett. 81, 2800 (2002).

[28] A. Richardella, P. Roushan, S. Mack, B. Zhou, D. A. Huse, D. D. Awschalom, and A. Yazdani, Science 327, 665 (2010).

[29] M. Johnson and R. H. Silsbee, Phys. Rev. Lett. 55, 1790 (1985).

[30] For extracting bias dependent $P_{\text {inj }}$, we assume that $P_{\text {inj }} \approx P_{\text {det }}$ at low bias $\left(V_{3 \mathrm{~T}}<0.05 \mathrm{~V}\right)$ and that the value of $P_{\text {det }}$ does not depend on the injector bias in the NL configuration.

[31] This prediction is based on Eq. (1) using the parameters extracted from NL measurements and assuming $P_{\operatorname{det}}$ depends on $V_{3 \mathrm{~T}}$ in the same manner as $P_{\text {inj }}\left(P_{\text {inj }}=P_{\text {det }}=\right.$ TSP $)$. While both $P_{\text {inj }}$ and $P_{\text {det }}$ drop with increasing bias in the 3T configuration, only $P_{\text {inj }}$ drops with increasing bias in the NL configuration. Hence, the calculated $3 \mathrm{~T}$ signal drops more rapidly than the NL one.

[32] As discussed in Ref. [18], the full cancellation of drift and diffusion terms, responsible for the suppression of the signal for $I_{\mathrm{inj}}<0$, occurs only for perfect one-dimensional geometry. This is not necessarily true for two- and three-dimensional geometries, what can lead to a sign reversal.

[33] A. N. Chantis, K. D. Belashchenko, D. L. Smith, E. Y. Tsymbal, M. van Schilfgaarde, and R. C. Albers, Phys. Rev. Lett. 99, 196603 (2007).

[34] L. R. Fleet, K. Yoshida, H. Kobayashi, Y. Kaneko, S. Matsuzaka, Y. Ohno, H. Ohno, S. Honda, J. Inoue, and A. Hirohata, Phys. Rev. B 87, 024401 (2013).

[35] J. Moser, M. Zenger, C. Gerl, D. Schuh, R. Meier, P. Chen, G Bayreuther, W. Wegscheider, D. Weiss, C.-H. Lai, R.-T. Huang, M. Kosuth, and H. Ebert, Appl. Phys. Lett. 89, 162106 (2006).

[36] M. Ishikawa, H. Sugiyama, T. Inokuch, K. Hamaya, and Y. Saito, Appl. Phys. Lett. 100, 252404 (2012).

[37] S. Sharma, A. Spiesser, S. P. Dash, S. Iba, S. Watanabe, B. J. van Wees, H. Saito, S. Yuasa, and R. Jansen, arXiv:1211.4460.

[38] Y. Pu, J. Beardsley, P. M. Odenthal, A. G. Swartz, R. K. Kawakami, P. C. Hammel, E. Johnston-Halperin, J. Sinova, and J. P. Pelz, Appl. Phys. Lett. 103, 012402 (2013). 